\title{
An experimental investigation simulating flow effects in first generation marine current energy converter arrays
}

\author{
L.E. Myers, A.S. Bahaj \\ Sustainable Energy Research Group, School of Civil Engineering and the \\ Environment, University of Southampton, Southampton, SO171BJ, UK \\ +0044 (0)2380593940 \\ L.E.Myers@soton.ac.uk \\ www.energy.soton.ac.uk
}

At present a small number of full-scale marine current energy converters are undergoing sea trials to demonstrate commercial viability of the technology. In order to provide meaningful quantities of electrical power to the grid, the next phase in the development of the technology will be the installation and operation of farms or arrays composed of multiple devices. As most tidal current sites are bi-directional and with bathymetry constraints, array layouts will necessarily take the form of highly optimized geometric configurations with reduced lateral inter-device spacing. This work discusses the concept of array layouts and proposes an appropriate and clear classification that can aid developers in understanding how arrays operate. This classification is supported by experimental studies conducted using several arrangements of multiple actuator disks to simulate early generation marine current energy converter arrays. The work presents quantification of the flow field around a 2-row array, device/device interaction as well as a study of the structure of the far wake region where subsequent devices could be installed. The results highlighted an optimal lateral spacing between devices where, under certain conditions flow can be accelerated between a pair of rotor disks. For the work presented here this accelerated region of flow possessed $22 \%$ more kinetic energy than the flow far upstream with no measurable negative effect upon the 2 actuator disks. This enhanced flow speed gives rise to the counterintuitive notion of a downstream row of devices producing more power than the upstream row.

Keywords: marine current energy converters, wake, array, flow acceleration

Abbreviations

ADV; Acoustic Doppler Velocimeter 


\section{Introduction}

Marine current energy conversion technology is presently at the commercial demonstrator stage where single devices of appreciable scale are operating at various tidal current sites around the world. Evidence of large amounts of available power at various European sites has long been known [1] with significant flow velocities measured around the British Isles, Philippines and far East, North East coast of North America and the West coast of Canada. There have been a number of studies conducted to attempt to

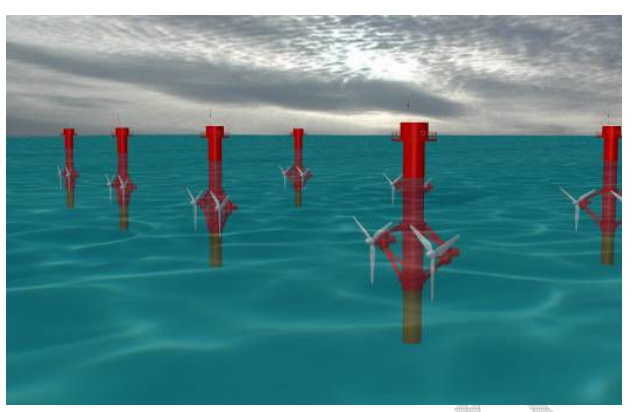

Figure 1. Artist's impression of an MCEC array. quantify the fraction of extractable energy from generic and specific sites using a variety of methods for analysis [2-5].

The next stage in the technology development path will be the installation of farms or arrays of Marine Current Energy Converters (MCECs) (Figure 1). The layout of such arrays may well be site dependant as a number of variables will determine interdevice spacing. Many sites have bi-directional flow characteristics usually associated with tidal currents, sometimes referred to as tidal streams or marine currents. Therefore, carefully designed geometric patterns may proliferate for the layout governing optimised arrangement of devices within an array. Installation and maintenance vessels will vary in size and manoeuvrability thus affecting inter-device spacing. In addition, the flow field generated by the MCEC devices will affect one another in an array. Fluid passing through a horizontal axis MCEC will experience a reduction in velocity across the rotor plane. Downstream of the rotor this region of fluid is moving at a lower velocity than the free stream fluid (that passed around the rotor) and hence must expand in order to conserve momentum. This takes the form of a gradually expanding cone-shaped region downstream of the rotor that is more commonly known as the wake. Turbulent mixing in the boundary region between the wake and the faster moving free stream fluid serves to re-energise the wake, breaking it up and increasing the velocity. At a distance far downstream the wake will have almost completely dissipated and the flow field will closely resemble that which existed upstream of the rotor disk.

Some of the variables that may influence the wake structure for a single rotor disk are illustrated in Figure 2. It will be a cost-benefit exercise for the developer of an array to decide at what lateral (cross flow) and longitudinal distances to separate MCECs; spaced too closely and device efficiencies will drop in the slower moving turbulent flow whilst over-spacing will not be an effective use of the tidal site which generally will have a compact spatial footprint. 


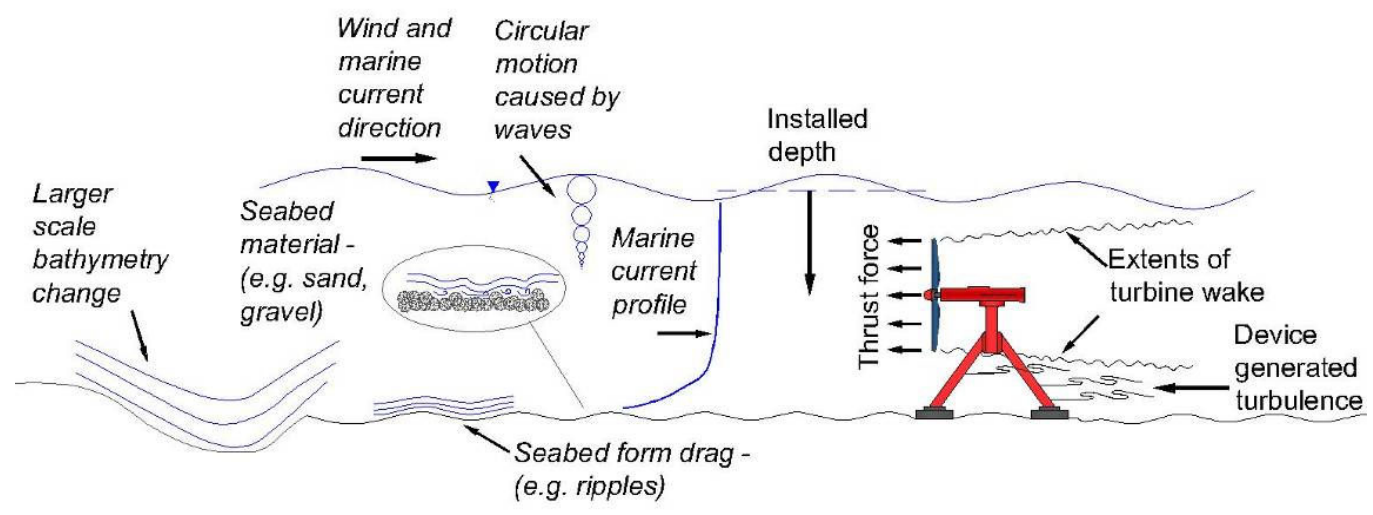

Figure 2. Variables affecting the flow field around MCECs.

The downstream wake generated by a MCEC will be significantly different to that of similar applications such as marine propellers or wind turbines. Principally due to the small total fluid height (rotor diameter may approach half total fluid depth), the pronounced velocity profile that exist in the sea and reduced flow re-energisation processes compared to the atmosphere. The principle variables that govern wake flow downstream of actuator disks in a vertically constrained flow were investigated by the authors and reported previously [6, 7].

There have been previous studies investigating the wake effects and energy losses within arrays of wind turbines $[8,9]$. Results have shown that energy losses due to interaction effects may be significant. This study establishes the case for marine currents turbines through experimental quantification of fluid around actuator disks used to simulate various turbine array configurations. The experimental studies described herein attempt to provide a seminal base for the investigation of interaction effect for multiple MCEC devices operating in a vertically constrained flow. The work characterises the flow field of two actuator disks aligned perpendicular to the flow with variable lateral spacing coupled with a comprehensive flow mapping experiment investigating an offset dual-row array of devices.

\section{Classification of MCEC arrays}

It is likely that arrays will evolve in size and complexity as the technology develops. A useful concept for classifying arrays has been developed by the authors as part of an EU-funded project aspiring to formulate protocols for equitable testing amongst wave and marine current energy devices [10]. A key driver for nearly all types of MCECs will be the minimisation of negative interaction effects between devices whereby structural loading is increased and/or power production is reduced. Early arrays are most likely to be composed of a single row of turbines aligned perpendicular to the incoming resource (where the resource has a low degree of directionality). Arrays can be expanded by including a second row where downstream devices are positioned in the spaces left between devices in the upstream row (see Figure 3). This is the limit of what we will refer to as $1^{\text {st }}$-generation arrays. This configuration has the following benefits.

a. It will minimise device interaction 
b. Maintenance and access to devices is not restricted as both rows can be approached from outside the array

c. Arrays can potentially become quite large with this configuration depending upon location

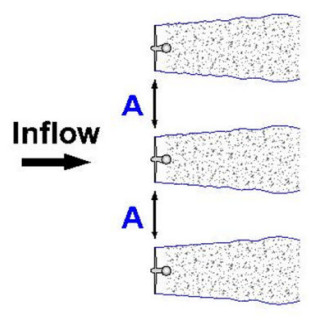

(a)

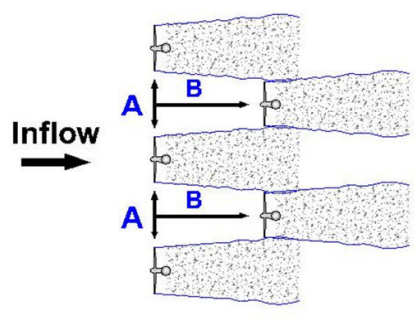

(b)

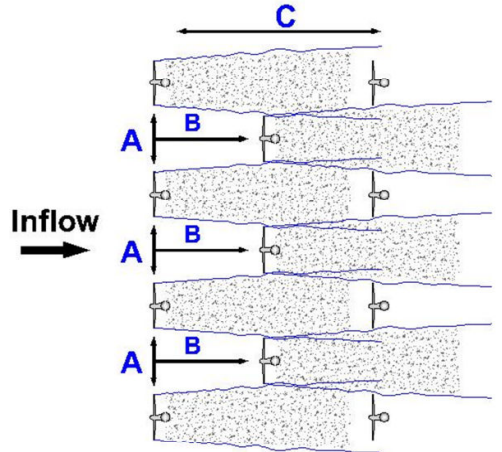

(c)

Figure $3.1^{\text {st }}$-generation arrays; single row (a), offset dual-row (b). $2^{\text {nd }}$-generation array (c)

Despite the present lack of experience at full-scale in the sea, it is possible to infer qualitative guidance for inter-device spacing within arrays. It is appropriate to prevent lateral spacing (dimension $A$ ) becoming too large within a single row array as it will not be an efficient use of space as most tidal energy sites are generally narrow. Wider arrays will also cause a larger obstruction to maritime traffic. With regard to general momentum theory it is well understood that very close spacing might lead to negative interaction effects such as reduced power capture or increased thrust forces. Therefore it follows that there must be an optimal value of $A$ for any situation. Ideally we would like a single row array to have good power capture performance whilst allowing good quality (at least equal velocity) flow through the gaps between devices in order to situate a second downstream row. Due to momentum theory the wakes of the upstream device must expand meaning that there will also be a corresponding optimum value of $B$.

Second generation arrays would be composed of 3 or more rows of devices where interaction effects are almost certain. Due to lateral spacing constraints (many tidal channels are narrow) it will be highly unlikely that unobstructed inflow could pass between 2 rows of devices hence the $3^{\text {rd }}$ row would be operating in the wake fields of upstream devices. The benefits of a large number of devices at the same site outweigh the potential for increased device loading and/or reduced performance and access issues for installation and maintenance to certain devices within the array. Figure 3 illustrates this issue as the furthest row downstream is most likely to encounter some form of negative interactive effects from the upstream rows whilst access to the middle row could be more difficult due to the bounding effect of the two adjacent rows. The distance $C$ is likely to be chosen using a cost/benefit approach. Closer spacing will allow a more compact array with greater energy capture per surface area but devices will be operating in more turbulent and slower flows. Increasing $C$ to a very large value will result in the opposite case. 
The descriptions given above means that the rated power of an array is independent of this classification. Instead it is driven by the operational complexity of the array. The classification of arrays in this manner is important as many of the device and performance metrics applied to arrays become more subjective for $2^{\text {nd }}$-generation arrays. Definition and comparisons between several $1^{\text {st }}$-generation arrays should, in theory, be easier.

\section{Experimental approach}

Modelling horizontal axis rotors becomes impractical at very small scale due to disparate scaling laws. Previous studies have shown that rotor diameters less than $0.8 \mathrm{~m}$ introduce problems due to the low incident energy flux available at flow speeds sufficiently low enough to avoid large Froude numbers and associated changes in water surface elevation [11]. Thus porous 'actuator' disks may be used at appropriate Froude numbers with scaled length ratios and low blockage ratios to exert a similar amount of thrust upon the fluid flow. Additional studies have also demonstrated that the far wake flow field generated by an actuator disk closely resembles that of a rotating horizontal axis turbine [12-14]. Flow will also be constrained between a number of actuator disks and mechanical rotors as both impart a similar thrust force upon the incident flow. This will be of use when studying multiple devices in close proximity.

As one might expect, full-scale Reynolds numbers for a tidal channel are high, in the order or $10^{7}$ and therefore fully turbulent. The transition to turbulent flow occurs at Reynolds numbers of approximately $2 \times 10^{3}$ and this was easily exceeded at the experimental facility used during this work.

In terms of device scaling all distances are scaled in a linear manner. The principle device scaling parameter is the amount of thrust force exerted on the fluid by the rotor. This is generally expressed as a non-dimensionless parameter, the thrust coefficient:

$$
C_{t}=\frac{\text { Thrust }}{0.5 \rho U_{0}^{2} A_{d}}=4 a(1-a)
$$

Where $\rho$ is the fluid density, $U_{0}$ is the free stream longitudinal flow velocity, $A_{d}$ is the area of the rotor and $a$ is the axial induction factor. The axial induction factor has a peak value of $1 / 3$ hence the optimum value of $C_{t}=0.9$. This equation is derived from actuator disk theory and is commonly found in wind turbine texts [15]. It applies equally to horizontal axis MCECs, the only difference being the fluid density and typical operating flow speeds. Thrust values for marine turbines per unit area are approximately 50 times greater than wind turbines (at typical operating flow speeds) and 5 times greater for typical rotor swept areas.

An Experimental programme was conducted at the tilting flume at the Chilworth research laboratory, University of Southampton. The flume is a conventional gravity fed flume with a working section $21 \mathrm{~m}$ in length, $1.35 \mathrm{~m}$ width and depths up to $0.5 \mathrm{~m}$. The vertical velocity profile in the flume is well developed [8,9] and closely resembles a modified $1 / 7^{\text {th }}$ power law with a more constant velocity close to the 
surface as measured at a full-scale tidal site [16]. Repeatability of flow velocities was in the order of $\pm 1 \%$ which was considered excellent for such a large open-channel flume. Turbulence intensity is in the range of $6-8 \%$ and is isotropic in nature due to the design of the inlet to the working section.

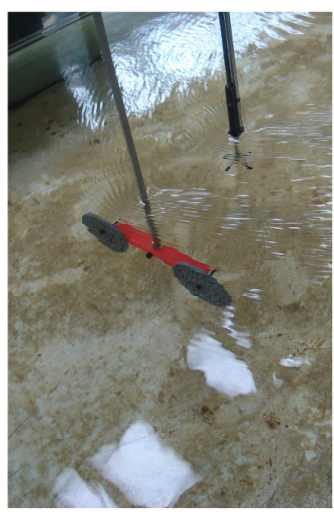

(a)

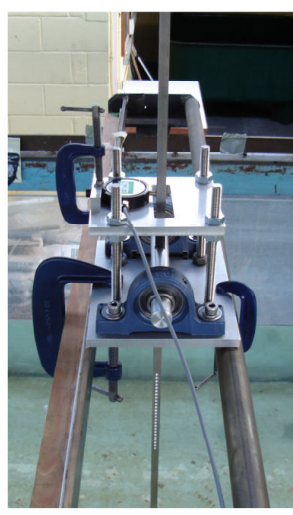

(b)

Figure 4. Dual disk mounted on a streamlined cross arm (a), thrust measurement rig (b)

Actuator disks of $100 \mathrm{~mm}$ diameter were mounted in the flow using a rig that incorporated a variable pivot arrangement to mechanically amplify the small thrust forces acting upon the disks (Figure 4). Measurements were taken with a $10 \mathrm{~N}$ button load cell mounted at the top of the rig with multiple sample periods recorded at each flow condition in order to ascertain the average thrust force. Disk porosities (ratio of open to closed area) were kept constant at 0.48 .

In order to visualise the flow field around the mesh disk rotor simulators a large number of point measurements were taken. A Nortek ADV device was used for high frequency velocity sampling in 3 directions. The uptake of such devices has been swift due to affordability and ease of use. The functionality and general accuracy of ADV devices has been addressed elsewhere [17-20]. The Vectrino ADV used for this work incorporated advanced firmware and was set to sample at $50 \mathrm{~Hz}$. The sample volume is cylindrical with a fixed diameter of $6 \mathrm{~mm}$. the volume height is user-defined and was chosen to be $3 \mathrm{~mm}$. Larger sample volumes will intercept more suspended matter in the water leading to stronger acoustic return signals and greater accuracy. However this can be negated as velocity shear between the top and bottom of the sample volume can lead to inaccuracies. Due to the high levels of suspended matter in the Chilworth flume, no doubt arising from being located in a hard water area, the signal strengths were found to be very strong. With the water depth set to $0.3 \mathrm{~m}$ the sample height represented $1 \%$ of the depth thus ensuring that velocity shear was minimal across measurement volume.

The inflow profile was measured upstream of the disk and a comprehensive set of downstream points were measured. Offset distances from the disk centre-axis were expressed in terms of disk diameters. Downstream measurements in the longitudinal axis generally extended from 3-20 diameters (D) and laterally (cross flume) up to 4D. For all tests presented the water depth was equal to $0.3 \mathrm{~m}$ or $3 \mathrm{D}$ which is similar to the ratio for present prototype and $1^{\text {st }}$ generation devices. Vertical measurement distance 
intervals were taken as $0.1 \mathrm{~d}$ where $\mathrm{d}$ is the total water depth. Each flow map consisted of 250-350 downstream point measurements depending upon the function of the experiment. The duration of each measurement was generally 180 -seconds which equated to 9000 discrete samples of velocity. This data set was then filtered according the method described by Cea et. al [21]. However due to the high concentration of suspended solids in the flume ADV acoustic signal strengths was consistently high, meaning that filtering only had a significant effect upon data accuracy when beam reflection errors occurred. These seldom occurred and could be mitigated by moving the ADV probe a few $\mathrm{mm}$ in the vertical direction. More details of filtering techniques and rationale can be found in [14].

\section{Data presentation}

Horizontal axis wake recovery can be defined in terms of velocity deficit, which is a non-dimensional number relative to the free-stream flow speed at hub height $\left(U_{0}\right)$ and the wake velocity $\left(U_{W}\right)$ :

$$
U_{\text {deficit }}=1-U_{w} / U_{0}
$$

Horizontal and vertical shear stresses are defined as:

$$
\begin{aligned}
& H S S=-\rho\left\langle u^{\prime} v^{\prime}>\right. \\
& V S S=-\rho\left\langle u^{\prime} w^{\prime}\right\rangle
\end{aligned}
$$

Where $u, v, w$ are the velocity components in the longitudinal (downstream), lateral (cross-channel) and vertical direction, $\rho$ is the fluid density and the dash denotes the varying component of the velocity (instantaneous velocity minus the sample mean velocity). Figure 5 shows the planes and axes of data presented in this paper.

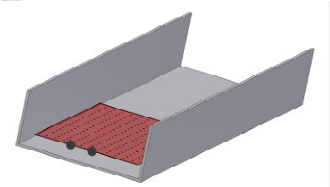

(a)

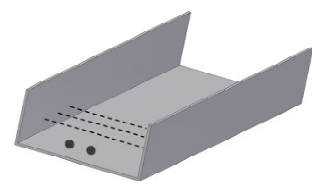

(c)

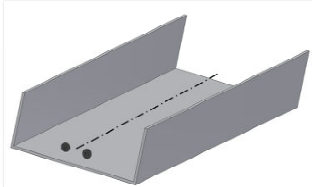

(b)

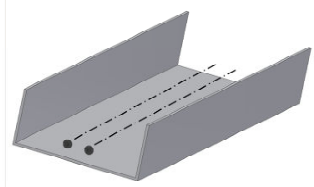

(d)

Figure 5. Diagrammatic illustration of experimental data presented herein: horizontal lateral plane (a), longitudinal centreline (b), lateral (c), longitudinal disk centreline (d). 


\section{Results and discussion}

\subsection{Lateral device spacing for single row tidal arrays}

For the first series of experiments a lateral array was considered with two actuator disks arranged perpendicular to the flow direction. Lateral disk separation, measured between the innermost edges of the actuator disks, was set to $0.5 \mathrm{D}, 1.0 \mathrm{D}$ and $1.5 \mathrm{D}$. Despite the good width of the Chilworth flume increasing the number of disks would increase the blockage area of this model-scale array to unrealistic values therefore only 2 disks were used. The $0.5 \mathrm{D}$ lateral separation case was based upon a commercial twin-rotor device presently in operation. Wider spacing was investigated as possible full-scale values that might be used within a $1^{\text {st }}$-generation single row array. It was assumed that spacing much in excess of $1.5 \mathrm{D}$ would mean that an array might be less efficient in terms of lateral coverage especially in more narrow tidal channels.

Disk thrust coefficients were found to vary with lateral separation despite the use of identical porous disks for all experiments. At 1D and 1.5D disk separation measured disk $\mathrm{C}_{\mathrm{T}}$ values were 0.91 consistent with previous single disk experiments at the same facility and water depth [14]. At $0.5 \mathrm{D}$ lateral separation individual disk $\mathrm{C}_{\mathrm{T}}$ values of 1.08 were measured. It is postulated that the high thrust force was due to the combination of pressure fields and the inability of the ambient flow to expand naturally around the inner edges of the actuator disks. This constriction of the stream tubes ability to expand increased the effective area of the disks thus increasing the thrust force compared to wider lateral spacing. Figure 6 shows a horizontal plane map of the u-direction (downstream) flow velocity for each of the three cases. Each plot is composed of approximately 150 point measurements. 


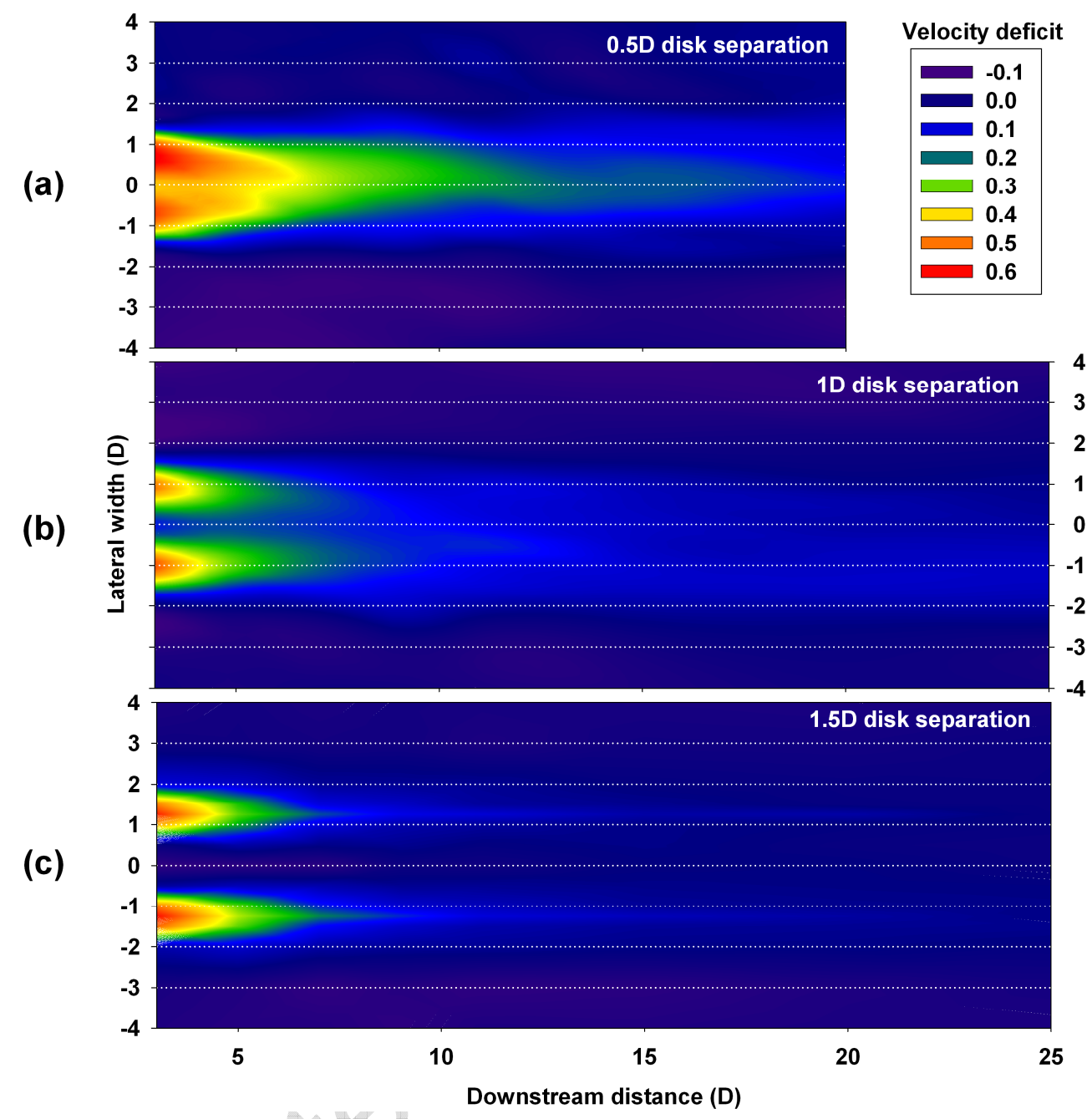

Figure 6. Horizontal lateral plane velocity flow fields around arrangements of twin actuator disks at varying lateral separation. (a) $0.5 \mathrm{D}$, (b) $1 \mathrm{D}$, (c) $1.5 \mathrm{D}$.

For the closest disk proximity $(0.5 \mathrm{D})$ the flow clearly merges in the near wake region less than 4-diameters downstream of the disks. In the far wake ( $>4 \mathrm{D}$ downstream) a singular wake with a Gaussian lateral profile persists far downstream from the 2 closely-spaced actuator disks, Fig 6 (a). At 1D lateral separation the flow downstream of each disk appears to remain separated in the near-wake region by relatively faster moving fluid between the actuator disks. Beyond 7D in the far wakes these two flow features merge into a singular wake, Fig.6 (b). At 1.5D lateral separation the wakes formed from each of the actuator disks are clearly separated by a central jet passing between the disks Fig.6 (c). The wakes do not merge and even in the very far wake it is possible to observe the centreline deficits from each disk. The central jet of fluid passing between the 2 disks moves at a greater velocity than the inflow to the 2-disk array demonstrating that it is possible to accelerate flow between 2 horizontal axis MCEC devices. This effect is more clearly shown in Figure 7. 


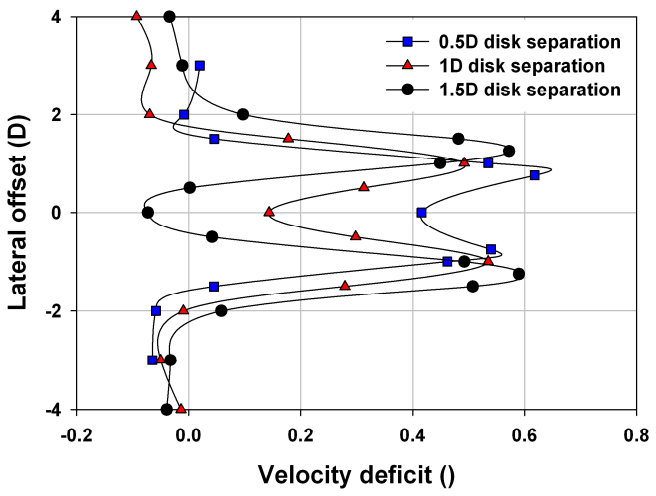

(a)

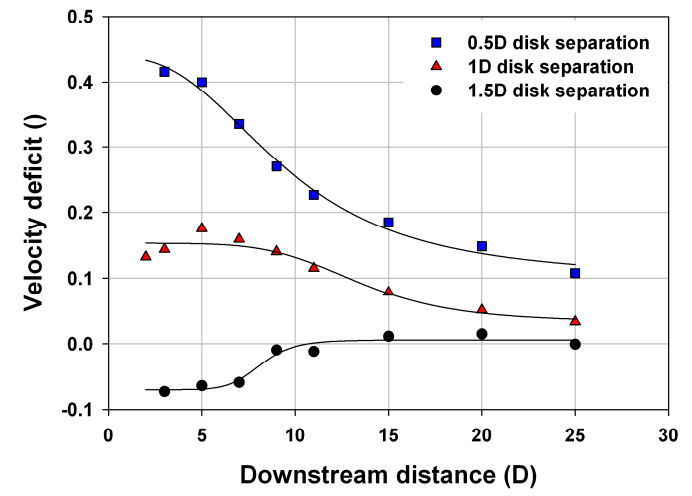

(b)

Figure 7. Velocity deficit plots of dual actuator disk arrangements; Lateral centre-depth at 3D

downstream (a) and longitudinal centreline (b)

The plot shown in Fig.7 (a) shows data for a lateral axis running across the flume at centre-depth (see Fig. 5c), 3-diameters downstream of the dual disk arrangements. The negative deficit existing for the 1.5D separation case demonstrates that flow has been accelerated between the disks. This region is approximately one diameter in width meaning that this is potentially exploitable for a device positioned downstream. In this case the velocity deficit at 3-diameters downstream is estimated to provide an increase in kinetic energy of the order of $22 \%$ compared to a device operating in free stream flow.

The longitudinal centreline plot (as Fig. 5b) shown in Fig.7 (b) also indicates that this faster moving jet of fluid persists up to $10 \mathrm{D}$ downstream with a relatively stable centreline velocity up to 6D downstream.

In must be noted that for smaller lateral separation distances less flow is channelled between the disks. In this case, this flow is also moving at a slower velocity than the inflow (illustrated by the positive velocity deficits, at $0.5 \mathrm{D}$ and 1D in Fig.7 (b)) and is thus not suitable for exploitation by a MCEC device.

\subsection{Offset 2-row arrays}

Following the creation of accelerated flow between a pair of actuator disks at $1.5 \mathrm{D}$ lateral separation a 2-row array configuration was tested. The downstream second row disk was placed 3-disk diameters downstream as shown in Figure 8. 

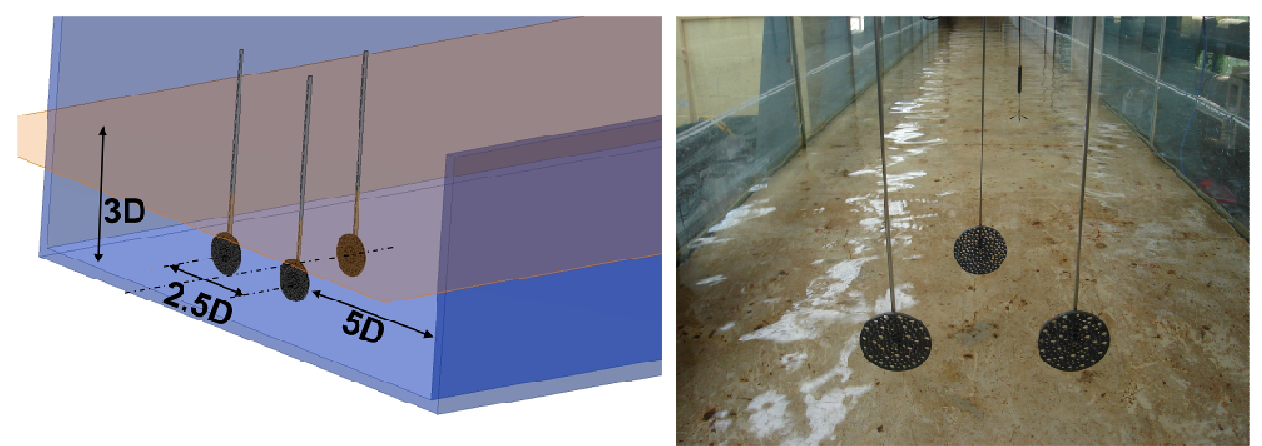

Figure 8. Arrangement of the 2-row array in the Chilworth circulating flume.

There are two issues arising from the 2-row offset configuration and the introduction of a downstream disk: (1) How the downstream disk might affect the flow conditions experienced by the upstream disks and (2) How the wakes formed by the upstream disks are affected by the presence of the downstream disk

Axial momentum theory states that half of the total momentum drop across an actuator disk occurs upstream. We also know that the distance over which this occurs is in the order of $0.5 \mathrm{D}$ thus it is not expected that the downstream second row disk, (in this case positioned 3-diameters downstream of the upstream pair), would have any effect. In all the experiment conducted, there was no observable change of the absolute thrust on the upstream, first row disks. That is $\mathrm{C}_{\mathrm{T}}$ remained at a value of 0.92. The position of the inflow velocity used to define $C_{T}$ was made $3 \mathrm{D}$ upstream from the disks but it could equally be taken further upstream or even at the disk location (in their absence) as the inflow did not vary with longitudinal distance.

The accepted methodology for defining inflow for wind turbines is a velocity measurement made at approximately 5-rotor diameters upwind. However, this cannot be applied for downstream devices in a MCEC array as will be demonstrated. The thrust coefficient for the downstream disk was calculated using the flow at the position of the disk in its absence. Unlike the 2 upstream actuator disks (first row) the downstream disk (second row) is positioned in a flow field that is varying in magnitude with longitudinal (and lateral) position and therefore measurement of inflow must be made at the disk position. However, using the method described to define the 'inflow' velocity the measured (non -dimensional) thrust coefficient was 0.91 , almost identical to the upstream pair of disks that had equal porosity.

Figure 9 shows the flow velocity in horizontal lateral plane of the 3-disk system. It was observed that the wakes of the upstream disks were influenced by the presence of the downstream disk. 
(a)

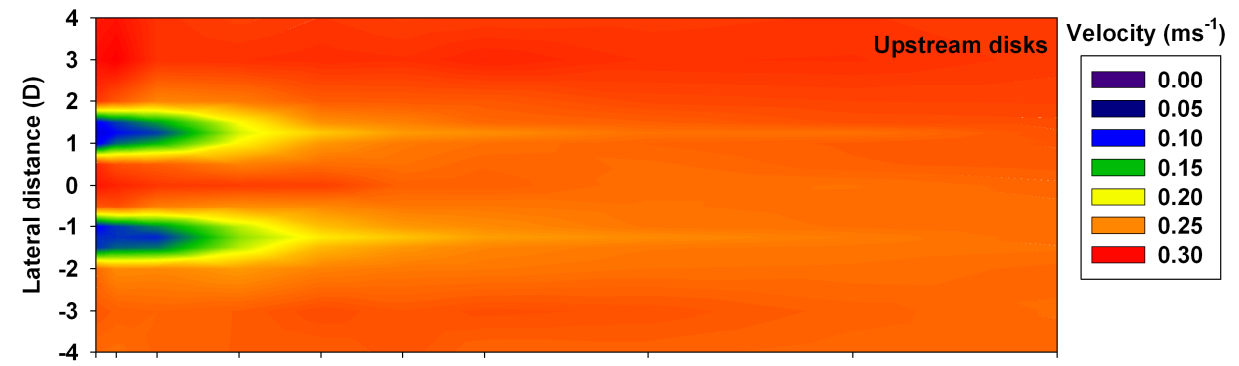

(b)

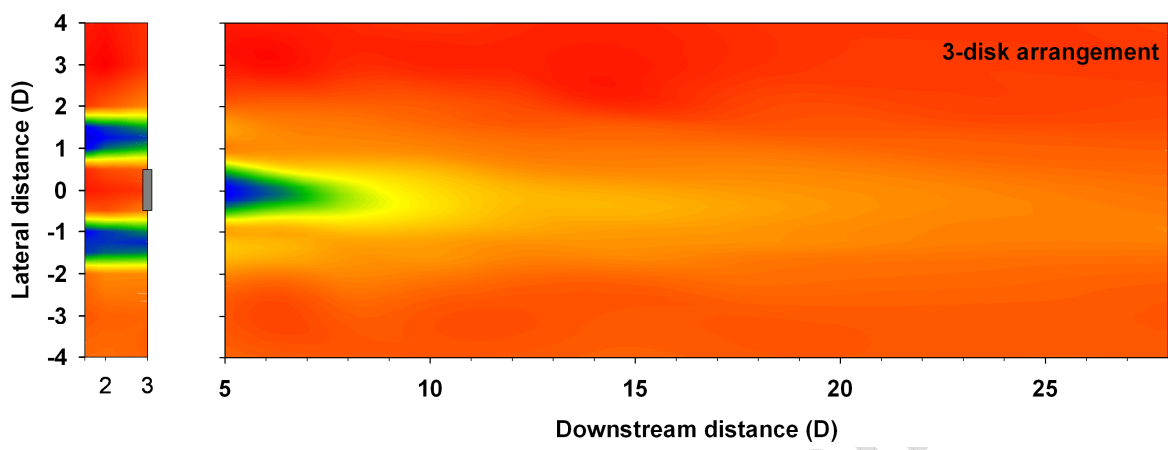

Figure 9. Horizontal lateral plane flow field velocity downstream of both (a) 2-disk and (b) 3-disk arrays.

Fig.9 (a) shows the velocity field for the 2 upstream first row disks in isolation (without the second row disk); this is essentially the base or ambient flow field for the downstream actuator disk. The plot in Fig.9 (b) shows the combined flow field of all 3 disks. Fig.9 (a) and Fig.9 (b) are aligned longitudinally for comparison with the location of the downstream disk shown diagrammatically. It is clear that the wakes formed by the 2 upstream disks merge with that emanating from the downstream disk. Between 5 and 7 diameters downstream of the upstream pair the wake intensity is much reduced; potentially diffused by the turbulence generated by the downstream disk. In the far wake a single wide wake can be observed.

The issue of the effect of the downstream disk upon the wakes formed by the upstream disks can be better observed by plotting lateral data downstream of the array. Figure 10 shows 4 lateral plots (as Fig. 5c) showing equivalent axes for both the upstream pair of disks and all 3 disks. Longitudinal distances downstream are relative to the upstream row. 

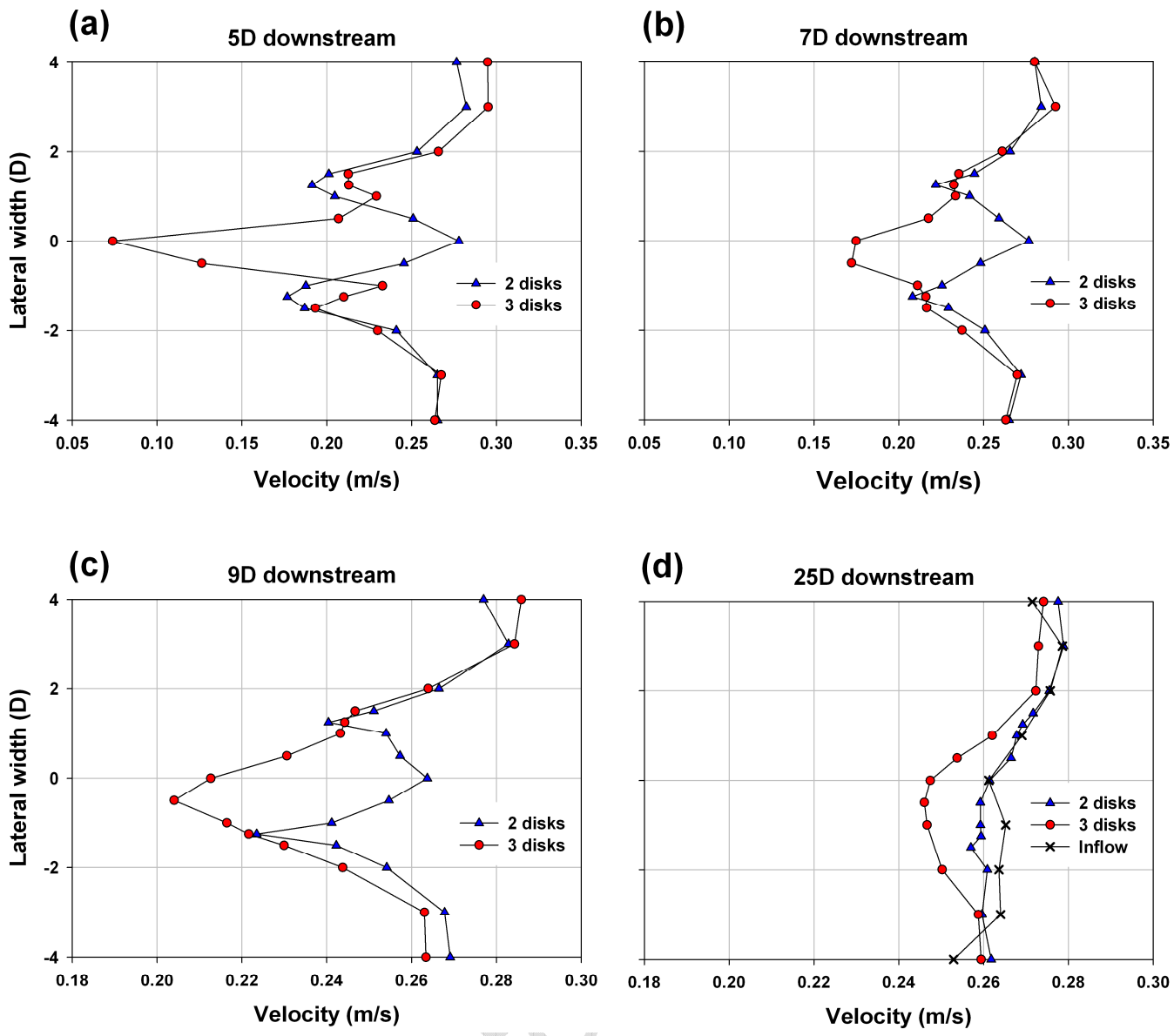

Figure 10. Lateral centre-depth velocity comparison between 2 and 3-disk arrays at increasing distance downstream

The inclusion of the downstream disk has a clear affect upon the upstream wakes at 5D downstream Fig10 (a). The centre of the upstream wakes is accelerated by the presence of the downstream disk and this is still apparent at 7D downstream Fig10 (b). The blockage-effect caused by the downstream disk will accelerate flow around its edge in a vertically constrained flow. Thus the centerline wake velocity of the upstream disks increases. In the same manner flow is accelerated between the flume side walls and the actuator disk array and can be seen the lateral extremities of the data presented in Fig.10 (c). The 3 wakes only fully merge at a point far downstream. The persistence of the wake generated by the 3-disk array is evident at 25D downstream. Fig.10 (d) The wake formed downstream of the 2-disk array has almost returned to the ambient condition no doubt aided by the accelerated flow between the disks and the separate wake structures offering an increased surface area over which shear forces can act to dissipate the wake flow. The 3-disk array does not share these features forming essentially 1 wide wake further downstream with a smaller surface area available for re-energisation and a deeper flow structure that is more difficult for the ambient flow to penetrate. It can also be observed that the far wake becomes skewed towards one side of the flume due to a non-uniform lateral flow distribution; a common occurrence in wide circulating channels, Fig10 (d). 
Figure 11 shows the non-dimensional velocity deficits for the individual actuator disks used in the 3 disk array (as Fig. 5d). All longitudinal distances are relative to the upstream pair of disks. As expected the centerline velocity deficit of the 2 upstream disks (without the inclusion of the downstream disk) are virtually identical. When the downstream disk is introduced it has a near wake velocity deficit slightly lower than the upstream pairing. This can be attributed to the increased turbulence and shear stress flowing into the downstream disk from the upstream pair.

In the far wake region the downstream disk has a velocity deficit that tends to the upstream pair if not a little higher. This could be explained by the reduction in influence from the wakes shed from the upstream disks. In the far wake the flow, whilst still slower than the ambient condition, has similar turbulence and shear stress characteristics (Figure 12). Thus the upstream wakes effectively shelter the downstream wake from ambient turbulence that serves to re-energise the wake flow of the downstream disk. Another area of interest is the change in upstream disk wake when the downstream disk is introduced (open circle points). Building upon the observations made around data shown in Figure 10 the upstream disk wake is accelerated around the downstream disk (caused by the pressure field and divergence of stream tubes). This is expressed as a lower velocity deficit in the region less than 10-diameters downstream. This effect then dissipates somewhat and in the far wake the centerline velocity deficit tends back towards the other cases.

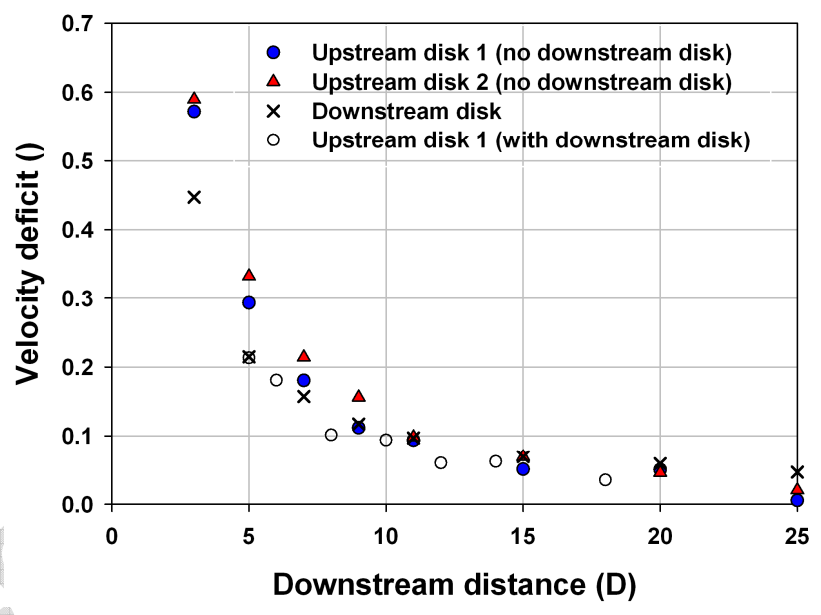

Figure 11. Longitudinal centreline velocity deficits for the actuator disk array

Figure 12 shows the horizontal shear stress downstream of the 2-disk first row arrangement which serves as the ambient inflow field to the second row disk situated downstream. The slight lack of longitudinal symmetry is a result of slightly greater flow velocity on the far-side of the channel. The shear stress is almost restored to the inflow condition at 11-diameters downstream. Thus we could expect a higher rate of wake recovery up to this point as the slower-moving fluid in the wake is broken down by the larger shear forces acting at its boundary. This hypothesis is upheld when inspecting the rate of wake recovery of the downstream second row disk in Figure 11. It shows a trend of faster recovery up to 10-diameters downstream but then the rate of recovery tends to that of the upstream disks. Velocity deficits in the far wake region for the 3-disk array are greater than the equivalent wake formed by a single disk [14] 
as the two-row, 3-disk wake is far wider and stronger meaning that ambient turbulence cannot penetrate to the wake centerline with such effectiveness.

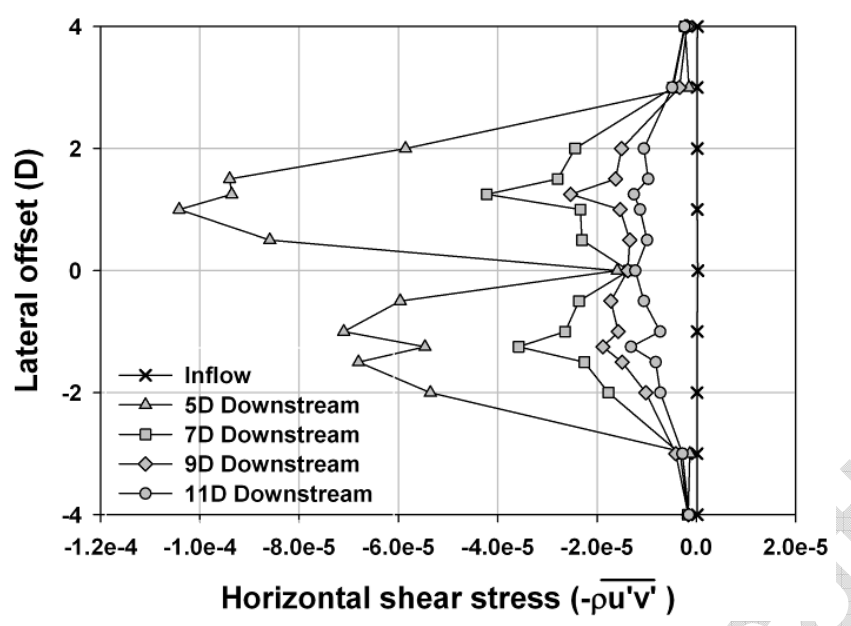

Figure 12. Lateral centre-depth horizontal shear stress downstream of 2-disk arrangement.

\section{Conclusions}

A new method of classifying MCEC arrays has been presented. This method is independent of both the number and type of devices and most importantly the total rated power of the array. Instead it is a function of operational complexity where later generation arrays require much greater levels of knowledge and experience in order to optimise performance.

A comprehensive flow mapping exercise around arrangements of multiple actuator disks has been presented. It has been demonstrated that flow can be accelerated between a pair of actuator disks arranged in a single-row $1^{\text {st }}$-generation array. For the dimensionless length ratios used in this work an inner disk separation of 1.5-diameters in a water depth of 3-diameters flow was accelerated between a pair of rotor disk simulators. The resulting jet between the disks had a greater velocity than the inflow resulting in an increased available kinetic energy of $22 \%$. This jet persisted downstream gradually reducing in velocity and lateral area.

For smaller values of lateral disk separation flow did pass between the pair of disks but with a lower velocity than the inflow condition. There is evidence from this study to suggest that the thrust to a pair of disks with a small lateral separation is increased due to the combination of pressure fields and the inability of the ambient flow to expand naturally around the inner edges of the rotor disks. This constriction of the stream tubes ability to expand increase the effective area of the disks thus increasing the thrust force compared to wider lateral spacing. It is anticipated that a study with horizontal axis mechanical rotors would confirm whether this increased thrust force would translate into an increase in power.

In summary for single row arrays the results show that: 
a) Close lateral separation increases the thrust force acting upon adjacent rotor disks

b) Optimum lateral separation can lead to accelerated flow passing between adjacent rotor disks with no negative impacts upon the disks

c) For the length ratios used in this study a jet was formed with a $22 \%$ increase in kinetic energy compared to the inflow to the actuator disks

d) The spatial extent of this jet will vary depending upon parameters such as: (i) lateral rotor disk separation, (ii) water depth, (iii) turbine rotor disk thrust (iv) inflow characteristics.

Following the work described above a 2 -row $1^{\text {st }}$-generation array was simulated experimentally. A third actuator disk was positioned along the flume (and disk) centreline 3-diameters downstream and situated in the strongest part of the accelerated jet of fluid. This disk was found to reduce the wake velocity deficits of the upstream disks in the immediate flow field. The wakes of the upstream disks were forced around the downstream device as would be expected for the flow around a single rotor disk. Thus reduced velocity deficits were measured extending beyond the near wake region up to 10-diameters downstream. Far downstream the wakes were found to be stronger compared to single disk arrangements. The combined wake formed downstream has an increased physical width reducing the ability of ambient flow to penetrate to the wake centreline. The lower surface area of the combined wake (compared to a wider-spaced 3 disk 2-row array arrangement) also lead to a lower level of wake dissipation in the far wake region. This may lead to increased longitudinal spacing for a third row of MCEC devices to enable interception of a flow velocity similar in magnitude to the first row. If this were to arise the increased power production from the devices in the second row should go someway towards offsetting any reduction in device density within the array.

It appears that from this work certain synergistic effects are possible within full-scale MCEC arrays whereby the power of a number of devices will be greater than an equivalent number of devices operating in isolation. Upstream devices can augment flow to a second downstream row with no negative repercussion upon the upstream devices. Dual-row arrays have the potential to generate tens of MW of power with the benefits of good access to all devices and little if any negative interaction effects.

In summary for the 2- row array studied the results show:

a) The downstream disk was subject to a greater thrust force than the upstream disks

b) The downstream disk did not affect the thrust acting upon the upstream disks

c) The near wake region of the upstream disks was deflected by the presence of the downstream disk.

d) The far wake region of the array had a higher velocity deficit (lower velocity) compared to a single disk due to the combined wake fields proving more difficult to break down

e) A third row of devices could be installed far downstream but in the shortmedium term wider 2-row arrays would offer a more favourable arrangement for a fixed number of devices within an array. 
Further experimental studies will be conducted to expand the results of this work with regard to investigating additional spatial and flow parameters in order to best inform the design of $1^{\text {st }}$-generation MCEC arrays.

\section{Acknowledgements}

This work was part of a project funded by the Department of Business, Enterprise and Regulatory Reform (now the Department of Business, Innovation and Skills) on performance characteristics and optimization of marine current energy converter arrays, BERR Project number T/06/00241/00/00. The work addressing classification of arrays is part of 'Equimar', funded under the European Community's Seventh Framework Programme FP7/2007-2013 under grant agreement number FP721338.

\section{References}

[1] European Commission, The exploitation of tidal marine currents. Report EUR16683EN, 1996.

[2] L.S. Blunden, A.S. Bahaj, Comparison of different approaches to site selection for tidal stream energy resource assessment. Proceedings 2nd International Conference of Renewable Energy in Maritime Island Climates (REMIC 2), Dublin, 26th - 28th April, pp. 139-144, 2006.

[3] L.E. Myers, A.S. Bahaj, Simulated electrical power potential harnessed by marine current turbine arrays in the Alderney Race. Renewable Energy, vol. 30, pp. 1713$1731,2005$.

[4] C. Garrett. P. Cummins, Limits to tidal current power. Renewable Energy, vol. 33, pp. 2485-2490, 2008.

[5] I.G. Bryden, S.J. Couch, How much energy can be extracted from moving water with a free surface: A question of importance in the field of tidal current energy? Renewable Energy, vol. 32, pp. 1961-1966, 2007.

[6] A.S. Bahaj, L.E. Myers, M.D. Thomson, N. Jorge, Characterising the wake of horizontal axis marine current turbines. Proceedings Seventh European Wave and Tidal Energy Conference, Porto, Portugal, 11-14 September 2007.

[7] L.E. Myers, A.S. Bahaj, R. Rawlinson-Smith, M. Thomson, The effect of boundary proximity upon the wake structure of horizontal axis marine current turbines. Proceedings 27th International Conference on Offshore Mechanics and Arctic Engineering, Estoril, Portugal, 15-20 June 2008.

[8] R.J. Barthelmie, Power losses due to wakes in large wind farms. Proceedings of the tenth World Renewable Energy congress, Glasgow, UK, $19^{\text {th }}-25^{\text {th }}$ July 2008.

[9] R.J. Barthelmie, S.T. Frandsen, M.N. Nielsen, S.C. Pryor, P.E Rethore, H.E. Jrgensen, Modelling and measurements of power losses and turbulence intensity in 
wind turbine wakes at middelgrunden offshore wind farm. Wind Energy, vol. 10, pp. 517-528, 2007.

[10] EquiMar, Funded by European Community's Seventh Framework Programme FP7/2007-2013 under grant agreement n FP721338, 2009-2011.

[11] L.E. Myers, A.S.Bahaj, Wake Studies of a 1/30th Scale Horizontal Axis Marine Current Turbine. Proceedings of the World Renewable Energy Congress, Regional meeting, Aberdeen, 22-27 May, 2005.

[12] P.J. Builtjes, The interaction of windmill wakes. Proceedings of the $2^{\text {nd }}$ Int. Symposium on wind energy systems, Amsterdam, 1978.

[13] P.M. Sforza, P. Sheerin, M. Smorto, Three-dimensional wakes of simulated wind turbines. AIAA Journal, vol. 19, pp. 1101-1107, 1981.

[14] L.E. Myers, A.S.Bahaj, Experimental analysis of the flow field around horizontal axis tidal turbines by use of scale mesh disk rotor simulators. Ocean Engineering, vol. 37, pp. 218-227, February 2010.

[15] T. Burton, D. Sharpe, N. Jenkins, E. Bossanyi, Wind Energy Handbook. John Wiley \& Sons, Ltd, 2001.

[16] UK Carbon Trust report, Europe and global tidal stream energy resource assessment. Black and Veatch consulting, 2005.

[17] A. Lohrmann, R. Cabrera, N.C. Kraus, Acoustic Doppler velocimeter (ADV) for laboratory use. Proceedings of the Symposium on Fundamentals and Advancements in Hydraulic Measurements and Experimentation, ASCE, pp. 351-365, 1994.

[18] G. Voulgaris, J.H. Trowbridge. Evaluation of the acoustic Doppler Velocimeter (ADV) for turbulence measurements. Journal of Atmospheric and Oceanic Technology, vol. 15, pp. 272-289, 1998.

[19] P.J. Rusello, A. Lohrmann, E. Siegel, T. Maddux, Improvements in acoustic doppler velocimetry. Proceedings of the Seventh International Conference on Hydroscience and Engineering, ICHE-2006, September 10-13, 2006.

[20] K. Blanckaert, U. Lemmin, Means of noise reduction in acoustic turbulence measurements. Journal of Hydraulic Research, vol. 44, pp. 3-17, 2006.

[21] L. Cea, J. Puertas, L. Pena, Velocity measurements on highly turbulent free surface flow using ADV. Experiments in Fluids, vol. 42, pp. 333-348, 2007. 